\title{
SECURE PRODUCTION OF FARM PRODUCE-ORIENTED MANAGEMENT AND SPATIAL DECISION-MAKING SYSTEM FOR PRODUCING AREA
}

\author{
Yan Wang ${ }^{1,2}$, Yuchun Pan ${ }^{2}$, Bojie Yan ${ }^{1,2}$, Anyun $\mathrm{Li}^{2,3}$, Jihua Wang ${ }^{2, *}$ \\ ${ }^{I}$ School of Geography, Beijing Normal University, Beijing, 100875, P. R. China \\ ${ }^{2}$ National Engineering Research Center for Information Technology in Agriculture, Beijing, \\ 100097, P. R. China \\ ${ }^{3}$ School of Information and Electrical Engineering, China Agricultural University, Beijing, \\ 100083, P. R. China \\ * Corresponding author, Address: National Engineering Research Center for Information \\ Technology in Agriculture, BanJing Road, HaiDian District, Beijing, 100097, P. R. China, \\ Tel: +86-10-51503488, Fax: +86-10-51503570, Email: wangjh@nercita.org.cn
}

Abstract: To address security of farm produce's quality, using object-oriented technologies to build multi-purpose database, it meets the current and future data needs of a variety of operational systems. Further, base on WebGIS, to establish management and decision-making system for producing area, This system could be implement digital management for the entire agricultural park, and evaluate individual plots momentarily, can also provide accurate results in the decision-making fertilizer.

Keywords: Secure production, spatial decision-making, WebGIS, multi-purpose database, environmental estimation for habitat

\section{INTRODUCTION}

Appropriate environment of habitats is infrastructure that be ensure the quality and safety of agricultural products, therefore, the level of safety of agricultural products can be fixed according to production environment. At present, with the rapid development of agriculture, agricultural chemicals,

Wang, Y., Pan, Y., Yan, B., Li, A. and Wang, J., 2008, in IFIP International Federation for Information Processing, Volume 259; Computer and Computing Technologies in Agriculture, Vol. 2; Daoliang Li; (Boston: Springer), pp. 909-916. 
such as fertilizers, pesticides, feed additives and others play a very important role, and cause agricultural pollution, soil productivity reduction and agroecological damage to the environment. Especially, the quality and safety of agricultural products, become the bottlenecks restraining continued efficient development in the new stage of agricultural production (Dai et al., 2002). Using spatial information technology to agricultural production for efficient management, and providing services for scientific decision-making about safe production of agricultural products, is the base to ensure efficient and safe agricultural production. Many scholars have begun to construct some intelligent management and decision-making systems using GIS technology. Moreover, they have applied to the actual production in a small area, and achieved certain results. But these databases only store a singlephase production data, thus making the decision-making is only reflecting status in quo, but the trend analysis of the habitats environment in a specific period, usefulness of the system has been greatly reduced; Furthermore, we only consider the evaluation of large field, divide the entire evaluation of the regional to grid directly, and evaluate by district, but not evaluate in accordance with the actual planting plot, this is detrimental to the overall agricultural park guide for effective operation of the plant. Current problems: database is static and can not reflect the real-time production and environmental conditions; Databases are designed using in only one system, and uniform standards and norms are not viable. Most systems are poor in data sharing and reuse.

This paper describes the design of a unified, scalable, multi-purpose database model. Based on it, we provide a management and decision support system based on WebGIS technology, which is good at updating database online, and using the real-time data for environmental assessment and appropriate decision-making. The entire decision-making is a dynamic process, not just for the historical inquiries of the decision-making result.

\section{MATERIALS AND METHODS}

\subsection{System Framework}

\subsubsection{Technology truss}

The design of system technology truss scheme is playing the significant role in the system design and application. In order to achieve the system target, this system adopts the B/S three development structural model. System collectivity technology truss as shown in Fig. 1. It contains client browser, Web server, GIS application server and database. 


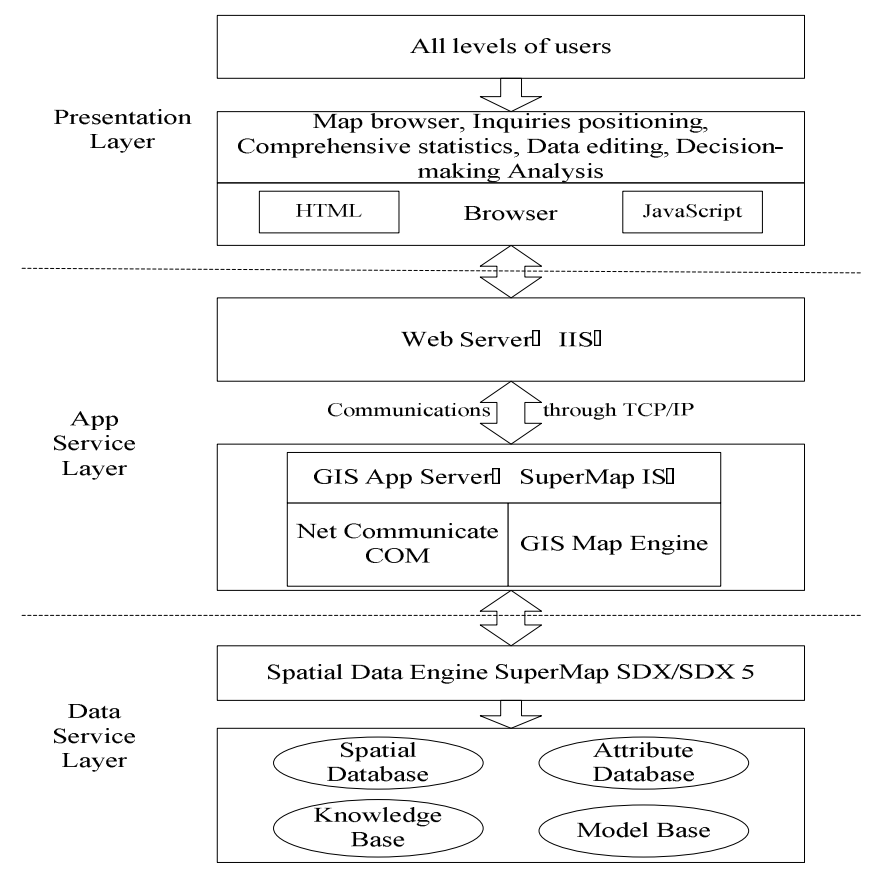

Fig. 1. System technology truss

The client browser supports a variety of data and information display and can communicate with the server. The Web server selects IIS (Internet Information Server), and is responsible for the basic network communication and the coordination. GIS server selects SuperMap IS, and mainly supports the realization of network geographic information system function. The Web server and GIS application server communicate through the Tcp/IP protocol (Ma et al., 2007). Use spatial data engine SuperMap SDX/SDX 5 to manage and operate special database, and to save the spatial data and the attribute data integrated to the database.

\subsubsection{Function structure}

The function structural design of this system divides into six big function modules. Information management module for plots and sampling points is foundation part of this system. The module mainly realizes map browser, spatial data and attribute data inquiry, statistical analysis as well as online edition. Online browser on spatial distribution of the attribute data is interpolation for the limited sampling point data in the land parcel scope, and obtaining the spatial distribution of soil nutrient or metal content, and the result can display in the form of thematic maps, also can display the value of random point on the map real time. Habitats environment appraisal module 
estimates the environment of plots, and grade to the land parcel by using space interpolation result and knowledge or the model relatively. Finally, display the analytic result, of what crop is fit for each plot. This module is easy to provide the basis of prenatal planning for the agricultural garden area. The decision-making module also gives fertilization prescription of each land parcel using the interpolation result and the decision-making model. This module is easy to scientific fertilization in production. The other two models mainly carry on the effective management to the decisionmaking knowledge, model as well as metadata.

\subsection{Design of Multi-purpose Database Model}

Information Sharing and Interoperability is the current field of information need to solve the hot issues. By design a multi-purpose database, the database will not only meet safety production of agricultural digital management needs, but also provide excellent data interface to the green or pollution-free agricultural habitats, safe production file management (IC card), tracking the quality and safety of agricultural products and other related business systems. For example, for green or pollution-free agricultural habitats declare operational systems, the system can provide the necessary testing of soil, water or nutrients metal elements, and can show grading data in plots of various products in different periods directly. Wherefore, the construction of multi-purpose database effectively improve the utilization of resources is also to avoid the duplication of database construction (Luo, 2006).

For building a multi-purpose database: firstly, it is necessary to standardize the data, in particular from the different departments, a need to formulate a unified standards for the corresponding standardized in order to achieve multi-source data integration and information sharing. The main problems of data standardization in the database design are as follow: the time consistency between space data and attribute data, the consistency of metric units, the consistency of data classification standard, the consistency of each data file for each number and each data field name.

Secondly, the establishment of the database metadata is also an important means of a multi-purpose database. This article metadata, including data collector, access time, content, themes, and the conversion of various operational information description and so on. For example, the users of space data can quickly understand the data names, scale, coordinate system and other describe information by metadata, so that different users, especially in Web environment could use the available data from heterogeneous platform expediently. 


\subsection{Decision-making Principle and Implementation}

\subsubsection{Process of extracting decision-making information}

Information extracting is a very important part of the decision-making, which need certain data storage in database for the screening, operating, etc. Then, we can use knowledge directly or input into decision-making model (Li et al., 2006). Following is the data flow diagram of the decision-making system (Fig. 2). The figure can be divided into two parts: inquiries sampling point and access plot data distribution.

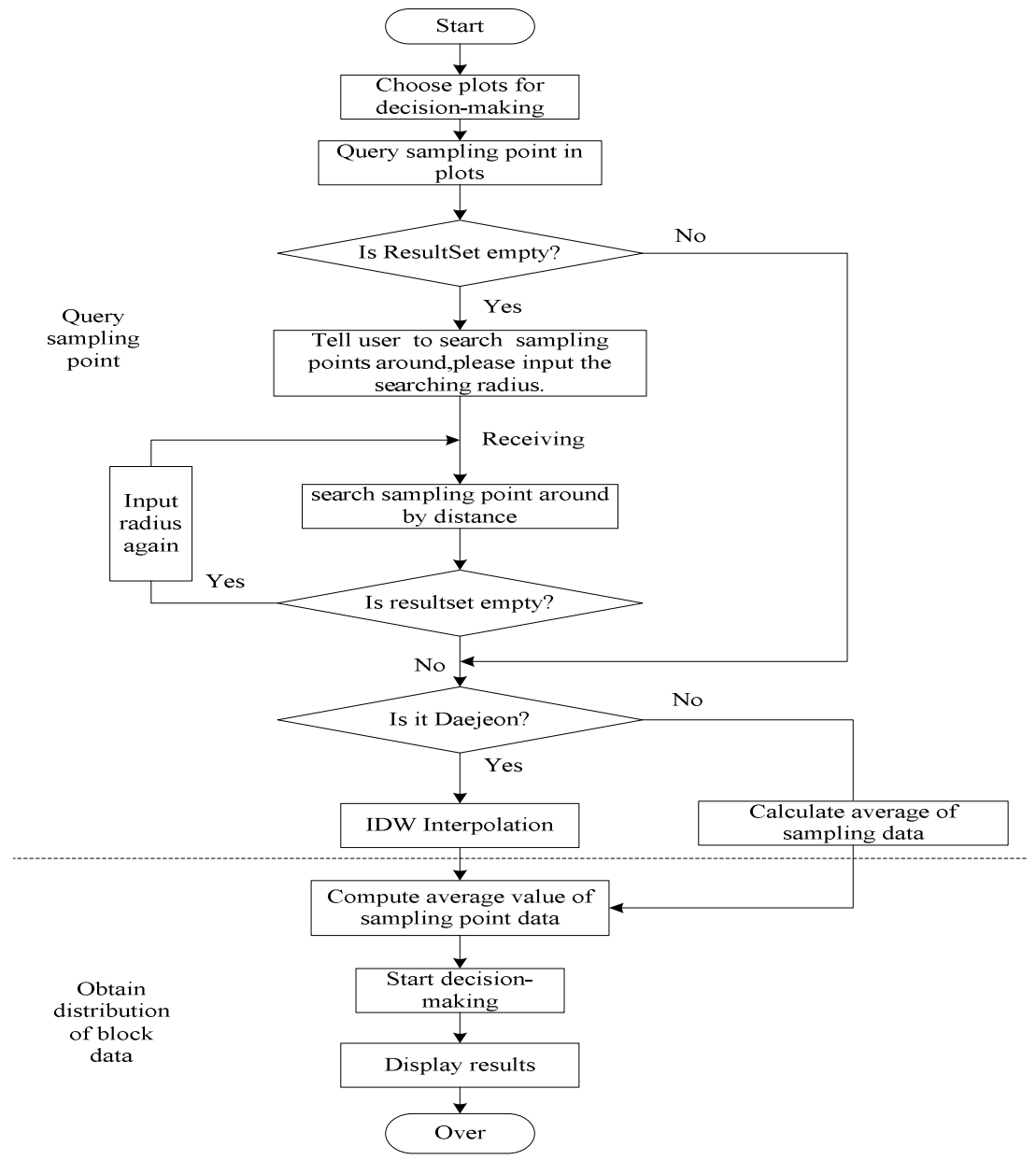

Fig. 2. Process of extracting decision-making information

(1) Inquiries data of sampling points

The decision-making system is targeted at farmland plot, and the decisionmaking data is sampling data. We need calculate the value of the entire plot through sampling data, and then could get the results of decisions based on 
the decision-making model. For some objective reasons, it is impossible for every plot has a sampling distribution, and consequently, there are some plots sampling, and some plots were not. For the former, we can use the plots directly within the sampling plot for decision-making, and for the latter, we need to search around the plot from the sampling points for decision-making. The paper describes the Map Control Methods of SuperMap, that finding plot away from the center within a certain range of other sampling sites.

(2) Inquiries data of plot distribution

In the forward step we got the sampling data, and on the following, we need to compute plot data through point data. In the agricultural park, the plot of farmland can be divided into two categories by size. Daejeon and so on are large one. And agriculture facilities (greenhouse or shed) is smaller, usually 1-2 acres.

To the large plot of this paper, we chose inverse distance weighted interpolation method to get the spatial distribution by the decision-making data.

To the small land, due to smaller area, various elements have a relatively small spatial variability. Therefore, we suppose the entire plot internal homogenization, and the paper directly calculates average value of all sampling points as value of the entire plot.

Thus, as the value of decision-making plot the spatial distribution of obtaining a dicision before the information extraction has been accomplished.

\subsubsection{Implementation of the decision-making}

We generalize all the optional form of decision-making in our system. We can make overall decision directly, analyzing integrated quality level of habitats environment. We also could make decision by subentry, only inspecting one of environmental soil, irrigation water or the atmosphere. In subentry decision-making, we can also evaluate heavy metal or nutrient content values individually. We study a certain substances such as $\mathrm{Pb}$ content exceeding whether or all the items, such as $\mathrm{Cu}, \mathrm{Cr}, \mathrm{Cd}$, get quality integrated of plot soil, irrigation water or air, and give appropriate decisionmaking results. In addition, each decision can be only on individual plots, but also on multiple plot decisions (Liu et al., 2006).

\section{RESULTS}

We develop the platform with the aid of Visual Studio.NET 2003, and use the C\# language to complete the system development, select the SuperMap 
IS.net specifically and the user-defined engine and C\# development pattern, and use SQL Server 2000 as database.

This system realizes the main function that has illuminated before. The core function is the environmental estimation for habitat and the suitability decision-making. Fig. 3 is result chart of many land parcel decision analysis.

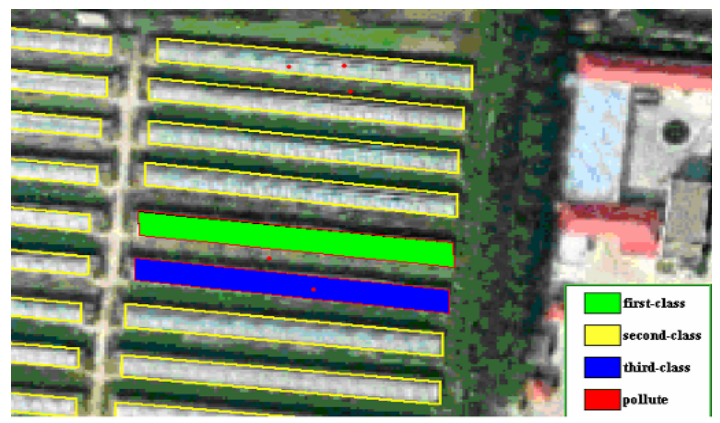

Fig. 3. Plots environmental assessment

The result of many land parcel decision-making demonstrates in form of the thematic maps. In the chart the green and the blue are two land parcels which be estimated. Rest on legend of the lower right corner. The green express that soil quality of the plot is first level. The first level reaches standard that protect region natural ecology and maintain natural background. It is optimal soil quality grading. Blue is third level. The third level are the minimum standard to guarantee the production of agriculture and forestry, and the normal growth of plant. Moreover, this module may carry on the single item appraisal for certain elements to inspect some elements specifically whether exceeding the allowed range. Thus, the policymakers of agricultural garden area may carry on planning before the production based on the appraisal result for the land parcel, and consider which land parcels should be plant which kind of crop. Moreover, they may consider adopting what measure to resume the land parcel already had be polluted, and may use fertilizer decision-making function to obtain precise fertilization prescription of each land in concrete production.

\section{DISCUSSION}

This paper proposes to construct management and decision-making system for farm produce habitat by using the WebGIS technology. First, it adopts the object-oriented technology to establish unified, extensible and the multi-purpose database. This database can not only satisfy the need of Secure Production of Farm Produce-Oriented Management, but also provide the very good data interface for declaration of the green or pollution-free production base, the production secure-oriented records management (the IC card), safe tracking quality of farm produce operational systems correlatively 
and so on. In the foundation, this database can manage the policy-making knowledge and model through constructing the knowledge library and the model library. Second, using SuperMap IS.NET as GIS development platform, it has realized basic functions, such as map browsing and inquiry, moreover realized real-time remote online data update. Thus it has guaranteed that the habitat decision-making is dynamic and real-time. Finally, the most core of the system is carrying on the environment appraisal and the suitability decision-making to the agricultural product habitats. So the system can provide scientific guidance for pre-natal planning of the agricultural garden area. Function of this system is not very perfect. For example, Historical data can be used for years of trend analysis. These will gradually complete in future. We will seek to establish a more perfect management and decision-making system to guarantee safe production of the farm produce.

\section{ACKNOWLEDGEMENTS}

This work was subsidized by Beijing Natural Science Fund (4061002), the program from Ministry of Agriculture (2006-G63) and the National High Tech R\&D Program of China (2006AA10Z201, 2007AA10Z203). The authors thank all teachers and students for their helpful cooperation in obtaining data documents and guiding of programming.

\section{REFERENCES}

Dai Xiaofeng, Zhao Bingqiang, Present State on Development of Safe Production Technology of China's Agricultural Products and Its Priority Field, Science and Technology Review, No. 3, 2002, pp. 46.

Ma Jin-feng, Pan Yu-chun, Shen Tao, Spatial decision support system for controlling the outbreak and spread of animal epidemics, Computer Applications, Vol. 27, No. 5, 2007, pp. 1289-1292.

Luo Ming-yun, Designing and Applying of a Soil Database System of Nanchong District Based on GIS, Chinese Journal of Soil Science, Vol. 37, No. 1, 2006, pp. 61-63.

Li Weijiang, Wu Yongxing, Mao Guofang, WebGIS-based information system for evaluation of soil environment in prime farmland [J], Transactions of the CSAE, Vol.22, No.8, 2006, pp. 59-63.

Liu Xiaojun, Zhu Yan, Yao Xia, et al. WebGIS-based system for agricultural spatial information management and aided decision-making [J],Transactions of the CSAE, Vol. 22, No. 5, 2006, pp. 125-129. 